\title{
A CHARACTERIZATION OF INNER PRODUCT SPACES
}

\author{
R. A. TAPIA
}

\begin{abstract}
In this paper we define a generalized inner product on an arbitrary normed linear space and use this generalized inner product to characterize inner product spaces in the class of all normed linear spaces. We give a sharp statement of a generalized Riesz representation theorem for bounded linear functionals. This theorem should be useful in generalizing the notions of gradient methods and reproducing kernel spaces.
\end{abstract}

1. Main result. We first prove the results stated in [12]. Let $X$ be a normed linear space and $f$ a functional defined on $X$. Recall that by the first right-hand Gâteaux derivative of $f$ at $x$ in the direction $h$ we mean

$$
f_{+}^{\prime}(x)(h)=\lim _{t \rightarrow+0} t^{-1}[f(x+t h)-f(x)],
$$

with the obvious definition for $f_{-}^{\prime}(x)(h)$. When $f_{+}^{\prime}(x)(h)=f_{-}^{\prime}(x)(h)$ we say that $f$ is Gâteaux differentiable at $x$ in the direction $h$. Let $f(x)=\frac{1}{2}\|x\|^{2}$ and $\langle x, y\rangle=f_{+}^{\prime}(x)(y)$.

Proposition 1. Every normed linear space is a generalized inner product space in the sense that

(a) $\langle x, y\rangle$ is well defined.

(b) $\|x\|=\langle x, x\rangle^{1 / 2}$.

(c) $|\langle x, y\rangle| \leqq\|x\|\|y\|$ (Cauchy-Schwarz-Buniakovsky inequality).

(d) If $X$ is an inner product space with inner product $[x, y]$, then $\langle x, y\rangle=$ $[x, y]$.

Proof. For $x, y \in X$ and $t>0$ let $R(t, y)=t^{-1}(\|x+t y\|-\|x\|)$. From Mazur [9, p. 75] we have that

(i) $R(t, y)$ is nondecreasing in $t$, and

$$
\text { (ii) }-\|y\| \leqq R(t, y) \leqq\|y\| \text {. }
$$

It follows that $T(y)=\lim _{t \rightarrow+0} R(t, y)$ exists. Also

(3) $\langle x, y\rangle=\lim _{t \rightarrow+0} \frac{1}{2}(\|x+t y\|+\|x\|) \lim _{t \rightarrow+0} t^{-1}(\|x+t y\|-\|x\|)=\|x\| T(y)$.

Received by the editors April 2, 1973.

AMS (MOS) subject classifications (1970). Primary 46B99, 46C10.

Key words and phrases. Inner product, differentiability of the norm, Riesz representation theorem.

(c) American Mathematical Society 1973 
This establishes part (a) of the proposition. Part (c) follows from (3) and (ii) of (2). The remainder of the proof is straightforward.

REMARK. For an interesting result concerning the Cauchy-SchwarzBuniakovsky inequality see Zarantonello [14].

THEOREM 1. The following are equivalent:

(a) $X$ is an inner product space.

(b) $\langle x, y\rangle$ is linear in $x$.

(c) $\langle x, y\rangle$ is symmetric.

Proof. Obviously (a) implies (b). Let $[x, y]=\frac{1}{2}(f(x+y)-f(x-y))$. Suppose (b) holds. Given $x, y, h \in X$ let $g(t)=[x+t h, y]$. We have

$$
g_{+}^{\prime}(t)=\frac{1}{2}(\langle x+t h+y, h\rangle-\langle x+t h-y, h\rangle)
$$

and by linearity $g_{+}^{\prime}(t)=\langle y, h\rangle$. Now by McLeod's mean value theorem for one-sided variations [10] we have $g(1)-g(0)=s_{1} g_{+}^{\prime}\left(u_{1}\right)-s_{2} g_{+}^{\prime}\left(u_{2}\right)$, where $0<u_{1}, u_{2}<1$ and $s_{1}+s_{2}=1$. Hence $[x+h, y]-[x, y]=\langle y, h\rangle$. If we let $x=0$, then $[y, h]=\langle y, h\rangle$. This shows that (b) implies (c) since $[x, y]$ is symmetric.

In order to show (c) implies (a) we will need the fact that $\langle x, y\rangle$ is continuous in $y$. We have $T\left(y_{1}+y_{2}\right) \leqq T\left(y_{1}\right)+T\left(y_{2}\right)$ (see [9]); hence from (3) $\langle x, y\rangle$ is also subadditive in $y$. It follows that

Also

$$
\langle x, y+h\rangle-\langle x, y\rangle \leqq\langle x, h\rangle \leqq\|x\|\|h\| .
$$

$$
\langle x, y\rangle \leqq\langle x, y+h\rangle+\langle x,-h\rangle \leqq\langle x, y+h\rangle+\|x\|\|h\| ;
$$

hence $-\|x\|\|h\| \leqq\langle x, y+h\rangle-\langle x, y\rangle$. Combining these last two statements we have

$$
|\langle x, y+h\rangle-\langle x, y\rangle| \leqq\|x\|\|h\|
$$

which establishes the continuity of $\langle x, y\rangle$ in $y$. Now suppose (c) holds. Then $\langle x, y\rangle$ is continuous in both variables. We will show $\langle x, y\rangle$ is additive in the second variable. This result is known for two-sided variations. Consider $h_{1}$ and $h_{2} \in X$. Given $\varepsilon>0$ there exists $\delta>0$ such that

$$
\begin{aligned}
& \mid f_{+}^{\prime}(x)\left(h_{1}+h_{2}\right)-f_{+}^{\prime}(x)\left(h_{1}\right)-f_{+}^{\prime}(x)\left(h_{2}\right) \\
& \quad-t^{-1}\left(f\left(x+t h_{1}+t h_{2}\right)-f(x)\right) \\
& \quad+t^{-1}\left(f\left(x+t h_{1}\right)-f(x)\right)+t^{-1}\left(f\left(x+t h_{2}\right)-f(x)\right) \mid \leqq 3 \varepsilon \\
& \text { whenever } 0<t<\delta .
\end{aligned}
$$

Hence

$$
\begin{aligned}
& \left|f_{+}^{\prime}(x)\left(h_{1}+h_{2}\right)-f_{+}^{\prime}(x)\left(h_{1}\right)-f_{+}^{\prime}(x)\left(h_{2}\right)\right| \\
& \quad \leqq t^{-1}\left|f\left(x+t h_{1}+t h_{2}\right)-f\left(x+t h_{1}\right)-f\left(x+t h_{2}\right)+f(x)\right|+3 \varepsilon .
\end{aligned}
$$


Now using McLeod's mean value theorem [10] we have

$$
\begin{aligned}
\mid f(x+t & \left.t h_{1}+t h_{2}\right)-f\left(x+t h_{1}\right)-f\left(x+t h_{2}\right)+f(x) \mid \\
\leqq & s_{1} t\left|f_{+}^{\prime}\left(x+t h_{1}+u_{1} t h_{2}\right)\left(h_{2}\right)-f_{+}^{\prime}(x)\left(h_{2}\right)\right| \\
& +s_{2} t\left|f_{+}\left(x+t h_{1}+u_{2} t h_{2}\right)\left(h_{2}\right)-f_{+}^{\prime}(x)\left(h_{2}\right)\right| \\
& +s_{3} t\left|f_{+}^{\prime}(x)\left(h_{2}\right)-f_{+}^{\prime}\left(x+t u_{3} h_{2}\right)\left(h_{2}\right)\right| \\
& +s_{4} t\left|f_{+}^{\prime}(x)\left(h_{2}\right)-f_{+}^{\prime}\left(x+t u_{4} h_{2}\right)\left(h_{2}\right)\right| \leqq 4 t \varepsilon \quad\left(0 \leqq u_{i}, s_{i} \leqq 1\right)
\end{aligned}
$$

if $t$ is sufficiently small. It follows that

$$
\left|f_{+}^{\prime}(x)\left(h_{1}+h_{2}\right)-f_{+}^{\prime}(x)\left(h_{1}\right)-f_{+}^{\prime}(x)\left(h_{2}\right)\right| \leqq 7 \varepsilon .
$$

This shows that $f_{+}^{\prime}(x)(y)$ is additive in $y$. Clearly $f_{+}^{\prime}(x)(a y)=a f_{+}^{\prime}(x)(y)$ if $a \geqq 0$. Now, if $a<0$, then $0=f_{+}^{\prime}(x)(a y-a y)=f_{+}^{\prime}(x)(a y)-a f_{+}^{\prime}(x)(y)$; hence $f_{+}^{\prime}(x)(y)$ is linear in $y$. It follows that $\langle x, y\rangle$ is a symmetric bounded bilinear form. Moreover from Proposition 1 it is positive definite. This proves the theorem.

REMARK. It has been pointed out to the author that once the continuity of $\langle x, y\rangle$ in $y$ has been demonstrated it is possible to show that (c) implies (a) using a result due to Alexandroff which says that in any finitedimensional normed linear space the points where the unit ball is smooth form a dense subset of the boundary of the unit ball. However, we prefer the direct proof given above.

2. Further properties of the generalized inner product. By the second right-hand Gâteaux derivative of $f$ at $x$ in the directions $h_{1}$ and $h_{2}$ we mean

$$
f_{+}^{\prime \prime}(x)\left(h_{1}, h_{2}\right)=\lim _{t \rightarrow+0} t^{-1}\left(f_{+}^{\prime}\left(x+t h_{1}\right)\left(h_{2}\right)-f_{+}^{\prime}(x)\left(h_{2}\right)\right) .
$$

Proposition 2. $\langle x, y\rangle=f_{+}^{\prime \prime}(0)(x, y)$.

Proof. A straightforward use of the definitions allows us to establish

$$
\langle a x, y\rangle=a^{2}\langle x, y \mid a\rangle, \text { for } a \neq 0 .
$$

Hence, since $\langle x, y\rangle$ is positively homogeneous in $y$, it must also be positively homogeneous in $x$. Therefore, from the definitions,

$$
f_{+}^{\prime \prime}(0)(x, y)=\lim _{t \rightarrow+0} t^{-1}\langle t x, y\rangle=\langle x, y\rangle \text {. }
$$

COROLlaRY 1. $X$ is an inner product space if and only if the second right-hand Gateaux variation of $f$ at the origin is linear or symmetric (or if $f$ is twice Fréchet differentiable at the origin).

We denote the dual of $X$ by $X^{*}$. Recall that if for $x \in X$ we let $J(x)=$ $\left\{g \in X^{*}:\|g\|=\|x\|\right.$ and $\left.g(x)=\|x\|^{2}\right\}$, then $J: X \rightarrow 2^{X^{*}}$ is called the duality map on $X$. Recall further the following two definitions given by Cudia in [4]. 
Definition 1. The normed linear space $X$ is said to be weakly uniformly convex in each direction if given $\varepsilon>0$ and $g, h \in X^{*}$ with $\|g\|=$ $\|h\|=1$ there exists $\delta>0$ such that

$$
|g(x+y)|>2-\delta \Rightarrow|h(x-y)|<\varepsilon
$$

for all $x, y$ with $\|x\|=\|y\|=1$.

Definition 2. The normed linear space $X$ is said to be weakly uniformly convex if given $\varepsilon>0$ and $g \in X^{*}$ with $\|g\|=1$ there exists $\delta>0$ such that

$$
|g(x+y)|>2-\delta \Rightarrow\|x-y\|<\varepsilon
$$

for all $x, y$ with $\|x\|=\|y\|=1$.

When we say the norm on $X$ is Gâteaux differentiable we mean at all points except the origin. We say $J$ is single valued when $J(x)$ is a singleton set for all $x \in X$.

THEOREM 2. The following are equivalent:

(a) The norm on $X$ is Gâteaux differentiable.

(b) $\langle x, y\rangle$ is homogeneous in $x$.

(c) $\langle x, y\rangle$ is homogeneous in $y$.

(d) $\langle x, y\rangle$ is linear in $y$.

(e) $\langle x, y\rangle=J(x)(y)$.

(f) $J$ is single valued.

(g) $X^{*}$ is weakly uniformly convex in each direction.

Proof. Cudia [4] has shown that (a) $\Leftrightarrow(\mathrm{g})$. Mazur [9] has shown that $(\mathrm{a}) \Leftrightarrow(\mathrm{f})$. That $(\mathrm{a}) \Leftrightarrow(\mathrm{c})$ follows from the fact that $f_{+}^{\prime}(x)(y)=-f_{-}^{\prime}(x)(-y)$. The identity given in (4) shows that $(b) \Leftrightarrow(c)$. Since a subadditive and homogeneous functional must be linear, we have that $(\mathrm{c}) \Leftrightarrow(\mathrm{d})$. Now, if $\langle x, y\rangle$ is linear in $y$, then by Proposition $1\langle x, \cdot\rangle \in X^{*},\langle x, x\rangle=\|x\|^{2}$ and $\|\langle x, \cdot\rangle\|=\|x\|$; hence $\langle x, \cdot\rangle \in J(x)$. However (c) clearly holds, therefore (a) holds which in turn implies that (f) holds. This establishes (d) $\Leftrightarrow(e)$ and proves the theorem.

Proposition 3. If the norm on $X$ is Gâteaux differentiable and $X$ is strictly convex, then the following are equivalent:

(a) $|\langle x, y\rangle|=\|x\|\|y\|$.

(b) $x=a y$.

Proof. If $y \neq x$ is such that $\|y\|=\|x\|$ and $\langle x, y\rangle=\|x\|\|y\|$, then for $0<c<1$

$$
\begin{aligned}
\|x\|^{2} & =c\langle x, x\rangle+(1-c)\langle x, y\rangle \\
& =\langle x, c x+(1-c) y\rangle \leqq\|x\|\|c x+(1-c) y\|<\|x\|^{2} .
\end{aligned}
$$

This inequality proves the proposition. 
We now generalize the Riesz representation theorem in terms of the generalized inner product $\langle x, y\rangle$. Let $J_{*}$ denote the duality map on $X^{*}$ and let $i$ denote the canonical or natural embedding of $X$ into its second dual $X^{* *}$. Perhaps the most general approach is that taken by Golomb and Tapia [5] where to each $\delta \in X^{*}$ they associate the subset of $X$ given by $i^{-1} J_{*}(\delta)$. Clearly if $x_{\delta} \in i^{-1} J_{*}(\delta)$, then $\left\|x_{\delta}\right\|=\|\delta\|$ and $\delta\left(x_{\delta}\right)=\left\|x_{\delta}\right\|^{2}$. Kolomy in [7, p. 700] has given a somewhat less general representation theorem; however neither of these approaches possesses the true flavor of the Riesz representation theorem, namely both lack a representation of the form $\left\langle x_{\delta}, y\right\rangle=\delta(y)$, for all $y \in X$ (although both approaches are certainly related to the representation theorem given below; see also Aubin [1] and Lumer [8]).

TheOREM 3. Suppose $X$ is a Banach space. Then the "Riesz representation theorem" holds: Given $\delta \in X^{*}$ there exists $x_{\delta} \in X$ such that

$$
\left\|x_{\delta}\right\|=\|\delta\| \text { and }\left\langle x_{\delta}, y\right\rangle=\delta(y), \text { for all } y \in X
$$

if and only if $X$ is reflexive with a Gâteaux differentiable norm.

Furthermore $x_{\delta}$ is unique (and the mapping $\delta \rightarrow x_{\delta}$ is continuous from the norm topology on $X^{*}$ to the weak topology on $X$ ) if and only if $X$ is also strictly convex.

In addition the mapping $\delta \rightarrow x_{\delta}$ is also continuous from the norm topology on $X^{*}$ to the norm topology on $X$ if and only if $X$ is also weakly uniformly convex.

Proof. If (5) holds, then $\left\langle x_{\delta}, y\right\rangle$ is linear in $y$ and by Theorem 2 the norm on $X$ is necessarily Gâteaux differentiable. Moreover (5) implies that every functional in $X^{*}$ attains its supremum on the unit sphere in $X$; hence by a well-known theorem (Klee [6]) $X$ is necessarily reflexive.

If the norm on $X$ is Gâteaux differentiable, then $J$ is single valued and $i^{-1} J_{*}(\delta) \Leftrightarrow i(x) \in J_{*}(\delta) \Leftrightarrow i(x)(\delta)=\|\delta\|^{2}$ and $\|x\|=\|\delta\| \Leftrightarrow \delta(x)=\|x\|^{2}$ and $\|x\|=\|\delta\| \Leftrightarrow x \in J^{-1}(\delta)$. Moreover, since $X$ is reflexive $i^{-1} J_{*}(\delta)$ is not empty. Therefore, if $x_{\delta} \in J^{-1}(\delta)$, then $\left\|x_{\delta}\right\|=\|\delta\|$ and $\left\langle x_{\delta}, y\right\rangle=J\left(x_{\delta}\right)(y)=$ $\delta(y)$.

Now, $x_{\delta}$ is unique $\Leftrightarrow J_{*}$ is single valued $\Leftrightarrow J_{*}$ is single valued and continuous from the norm topology on $X^{*}$ to the weak* topology in $X^{* *} \Leftrightarrow X$ is weakly uniformly convex in each direction $\Leftrightarrow X$ is strictly convex (since $X$ is reflexive). Also $J_{*}$ is single valued and continuous from the norm topology on $X^{*}$ to the norm topology on $X^{* *} \Leftrightarrow X$ is weakly uniformly convex. For details see Cudia [4] and also Golomb and Tapia [5]. This proves the theorem.

The author would like to thank Dr. James McClellan for suggesting the proof of the fact that $\langle x, y\rangle$ is continuous in the $y$-variable. He also 
would like to thank Dr. Gilbert de Montricher and Professor B. Frank Jones for their comments on an earlier draft of this paper.

ADDED IN PROOF. Professor Laugwitz has pointed out to the author that the following papers are also related to the above work: D. Laugwitz and E. R. Lorch, Riemann metrics associated with convex bodies and normal spaces, Amer. J. Math. 78 (1956), 889-894. E. R. Lorch, A curvature study of convex bodies in Banach spaces, Ann. Mat. Pura Appl. 34 (1953), 105-112.

\section{REFERENCES}

1. J. P. Aubin, Optimal approximation and characterization of the error and stability functions in Banach spaces, J. Approximation Theory 3 (1970), 430-444. MR 43 \#795.

2. A. Beurling and A. E. Livingston, $A$ theorem on duality mappings in Banach spaces, Ark. Mat. 4 (1962), 405-411. MR 26 \#2851.

3. R. Bonic and F. Reis, A characterization of Hilbert space, An. Acad. Brasil Ci. 38 (1966), 239-241. MR 36 \#1961.

4. D. Cudia, The geometry of Banach spaces. Smoothness, Trans. Amer. Math. Soc. 110 (1964), 284-314. MR 29 \#446.

5. M. Golomb and R. A. Tapia, The metric gradient in normed linear spaces, Numer. Math. 20 (1972), 115-124.

6. V. L. Klee, Jr., Some characterizations of reflexivity, Rev. Ci. (Lima) 52 (1950), nos. 3-4, 15-23. MR 13, 250.

7. J. Kolomy, Some remarks on nonlinear functionals, Comment. Math. Univ. Carolinae 11 (1970), 693-704. MR 44 \#869.

8. G. Lumer, Semi-inner-product spaces, Trans. Amer. Math. Soc. 100 (1961), 29-43. MR 24 \#A2860.

9. S. Mazur, Über konvexe Mengen in linearen normierten Raumen, Studia Math. 4 (1933), 70-84.

10. R. McLeod, Mean value theorems for vector valued functions, Proc. Edinburgh Math. Soc. (2) 14 (1964-65), 197-209. MR 32 \#2522.

11. K. Sundaresan, Smooth Banach spaces, Math Ann. 173 (1967), 191-199. MR 36 \#1960.

12. R. A. Tapia, A characterization of inner product spaces, Bull. Amer. Math. Soc. 79 (1973), 530-531.

13. M. M. Vainnberg, Variational methods for the study of nonlinear operators, GITTL, Moscow, 1956; English transl., Holden-Day, San Francisco, Calif., 1964. MR 19, $567 ; 31$ \#638.

14. E. H. Zarantonello, The meaning of the Cauchy-Schwarz-Buniakovsky inequality, Mathematics Research Center Report 1277, University of Wisconsin, Madison, Wis., 1972.

Department of Mathematical Sciences, Rice University, Houston, Texas 77001 